\title{
Terahertz Time-Domain Spectroscopy of Raman Inactive Phonon-Polariton in Strontium Titanate
}

\author{
Seiji Kojima, ${ }^{\text {a, b) }}$ and Tatsuya Mori ${ }^{\text {b) }}$ \\ Division of Materials Science, University of Tsukuba, Tsukuba, Ibaraki 305-8573, \\ Japan \\ ${ }^{a}$ Corresponding author: kojima@bk.tsukuba.ac.jp \\ b) These authors contributed equally to this work.
}

\begin{abstract}
The Raman inactive low-frequency phonon-polariton was studied in a quantum paraelectric strontium titanate crystal. The lowest frequency IR active $\mathrm{T}_{1 \mathrm{u}}$ mode was investigated by the reflectance and transmittance Terahertz Time-Domain Spectroscopy using the pulse coherent $\mathrm{THz}$ radiation from 0.2 to 5.0 THz. The complex dispersion relations were determined using the real and imaginary parts of a polariton wavevector calculated from the complex refractive index which reflects the $\mathrm{T}_{1 \mathrm{u}}$ symmetry phonon-polariton. The remarkable change of the dispersion relation was observed in the vicinity of TO and LO mode frequencies for both real and imaginary parts of a polariton wave vector. The observed complex dispersion relation of $\mathrm{T}_{1 \mathrm{u}}$ symmetry is in agreement within the experimental uncertainty with the dispersion curves fitted by the damped harmonic oscillator model.
\end{abstract}

Keywords Ferroelectric; polariton; dispersion; soft mode; strontium titanate; THz-TDS 


\section{INTRODUCTION}

In general, IR active phonons couple with photons, and the mixture of phonon and photon was described as a coupled excitation of phonon-polariton [1,2]. The polariton dispersion relation with no damping was observed for the first time by the forward Raman scattering in a Gallium phosphide crystal [3]. The damping of polariton for the transverse mode was also discussed [4,5]. After that, the phonon-polariton dispersion relation has been extensively studied in many crystals. Quantum paraelectrics such as $\mathrm{SrTiO}_{3}, \mathrm{KTaO}_{3}$, have attracted much attention by the suppression of a ferroelectric phase transition down to $0 \mathrm{~K}$ [6]. In the paraelectric phase, IR active modes are Raman inactive, therefore, the observation of phonon-polariton by forward Raman scattering is impossible. Inoue et al. observed the polariton dispersion for the first time using hyper-Raman scattering technique [7]. However, by the conservation law among the wavevectors of incident, scattered light and polariton, the observation of polariton of the lowest frequency mode was impossible because of the difference of refractive indices between fundamental and second harmonic wavelengths.

The development of a stable femtosecond pulse laser has enabled the coherent $\mathrm{THz}$ radiation using a photoconductive antenna. The reliable unique determination of the real and imaginary parts of the dielectric constant can be possible by the THz-time domain spectroscopy (THz-TDS). Up to the present, the frequency range of the most THz-TDS studies was limited below $2 \mathrm{THz}$. However, very recently, the widely tunable monochromatic Cherenkov phase-matched $\mathrm{THz}$ wave generator was developed using nonlinear optic crystals of ferroelectric $\mathrm{MgO}$ doped $\mathrm{LiNbO}_{3}$ [8]. This enables the extension of the frequency limit of THz-TDS up to a higher frequency [9]. Since the study of the lowest frequency infrared active modes is very important for understanding the mechanism of ferroelectric instability, such a polar mode propagates as a polariton in a crystal. It was confirmed that THz-TDS is very useful to determine the polariton dispersion relation of a ferroelectric soft mode [10]. In the present study, we report the complex dispersion relation of phonon-polariton including the damping, which was reported for a strontium titanate crystal using the broadband THz-TDS.

\section{EXPERIMENTAL}

$\mathrm{A} \mathrm{SrTiO}_{3}$ single crystal purchased from Furuuchi Chemical Corp. with the size of 10 $\times 10 \times 0.5 \mathrm{~mm}^{3}$ was used for both reflectivity and transmittance measurements. 
Reflectivity spectra were measured using the THz-TDS system (Advantest TAS7500SU) with a standard reflection configuration at near normal incidence with the incidence angle of about $12^{\circ}$, using a high-speed asynchronous optical sampling technique. The scan rate and frequency resolution were $8 \mathrm{~ms}$ and $7.6 \mathrm{GHz}$, respectively. A Cherenkov-type $\mathrm{THz}$ pulse generator and photoconductive antenna were used as the emitter and detector, respectively, triggered by a femtosecond laser with a wavelength of $1560 \mathrm{~nm}$ and a pulse width of about $45 \mathrm{fs}$. The repetition rate of the femtosecond laser for the emitter was $50 \mathrm{MHz}$, and for the detector it was slightly modulated from $50 \mathrm{MHz}$. A polished aluminium mirror was used as a reference [11, 12]. THz transmission spectra were measured by using the THz-TDS system (Tochigi Nikon RT-10000) and low temperature-grown GaAs photoconductive antennas for both the emitter and detector. These antennas were triggered by a mode-locked Ti:sapphire laser with a wavelength of $780 \mathrm{~nm}$, a pulse width of about $100 \mathrm{fs}$, and a repetition rate of $80 \mathrm{MHz}[13]$.

In the normal analysis procedure of a reflectivity measurement, the following relation is used to extract the complex refractive index $\tilde{n}=n+i \kappa$,:

$$
\frac{\tilde{E}_{s a m}}{\widetilde{E}_{r e f}}=\frac{1-\tilde{n}}{1+\tilde{n}}
$$

where $\tilde{E}_{\text {sam }}$ and $\tilde{E}_{\text {ref }}$ are the amplitude spectra of the $\mathrm{THz}$ pulse reflected from the sample and aluminium reference, respectively. However, in THz-TDS reflectivity measurement, there is always a position misalignment of micrometers between the sample and the reference owing to the experimental error causing serious deviation from the original structure of the complex refractive index. In this study, we have corrected the experimental error originated from the position misalignment by the following procedure [12].

Firstly, we determine the complex refractive index $\tilde{n}_{\text {trans }}=n_{\text {trans }}+i \kappa_{\text {trans }}$ by a transmission configuration THz-TDS measurement. Secondly, using the observed $\tilde{n}_{\text {trans }}$, we calculate the transmitted phase shift $\phi(\omega)_{\text {trans }}$ of the complex reflection coefficient between vacuum and the sample by the following relation:

$$
\phi(\omega)_{\text {trans }}=\tan ^{-1} \frac{2 \kappa}{1-n^{2}-\kappa^{2}}
$$

Assuming $\phi(\omega)_{\text {trans }}$ as accurate, we fit the phase shift obtained from the reflectivity 
measurement to $\phi(\omega)_{\text {trans }}$ by shifting of the time-domain waveform, and we obtain the shifted time $\Delta t$. Finally, we determine the complex refractive index of $\mathrm{SrTiO}_{3}$ using the following relation:

$$
\frac{\widetilde{E}_{\text {sam }}}{\widetilde{E}_{\text {ref }}} e^{i \omega \Delta t}=\frac{1-\tilde{n}}{1+\tilde{n}}
$$

\section{POLARITON DISPERSION OF COMPLEX WAVE VECTOR}

When a photon is incident to a polar dielectric medium, it couples to polar optical phonons and propagates as phonon-polariton as shown in Fig. 1. According to Huang's analysis [2], the dispersion relation of polariton was given by the equation,

$$
\varepsilon(k, \omega)=\frac{c^{2} k(\omega)^{2}}{\omega^{2}}
$$

where $\omega, k$, c, and $\varepsilon(k, \omega)$ are angular frequency, wave vector, light velocity, and dielectric constant, respectively. Up to the present, the most of studies on phonon-polariton have discussed the dispersion relation assuming that $\varepsilon(k, \omega)$ is a real number, which means that the damping of polariton is negligible. The polariton dispersion relation with no damping was observed for the first time by Raman scattering on a GaP crystal, and the anti-crossing of polariton branches in the vicinity of $\mathrm{TO}$ and LO mode frequencies were clearly observed at small scattering angles below $10^{\circ}$ [3]. The damping of the lowest TO mode of GaP is negligible, therefore the polariton frequency vs. wave vector was discussed without consideration of the polariton damping. However, the damping factor of the soft mode of a ferroelectric is generally large, and the soft mode becomes overdamped in some ferroelectrics such as $\mathrm{BaTiO}_{3}$ and $\mathrm{KH}_{2} \mathrm{PO}_{4}[14,15]$. Therefore, for the study of the polariton dispersion of ferroelectric materials, to discuss damping using a complex dielectric constant is important.

When the dielectric constant, $\varepsilon(k, \omega)$, becomes complex, then the wave vector, $k$ or a frequency, $\omega$, can become also a complex number. Generally, in the IR spectroscopy considering spacial decay, $\omega$ is defined as a real number, while $k$ is defined as a complex number [4], and the polariton damping of the transverse mode with E perpendicular to the c-axis was discussed in polycrystalline $\mathrm{MgF}_{2}$ using comlex wavevector, $k$, for real frequency. The complex wavevector, $k$, is defined by the equation,

$$
k(\omega)=k^{\prime}(\omega)+\mathrm{i} k^{\prime \prime}(\omega) \text {. }
$$

Considering the following relation between the complex dielectric constant and the complex refractive index,

$$
\varepsilon(\mathrm{k}, \omega)=\{n(k, \omega)+\mathrm{i} \kappa(k, \omega)\}
$$

The polariton dispersion relation for the real and imaginary parts of the complex $k$ was 
derived from eqs. (1)-(3),

$$
k^{\prime}(\omega)=\omega n(k, \omega) / c, \quad k^{\prime \prime}(\omega)=\omega \kappa(k, \omega) / c \quad .
$$

In the damped harmonic oscillator model with a TO mode, the following equation holds.

$$
\frac{c^{2} k(\omega)^{2}}{\omega^{2}}=\varepsilon_{\infty}+\frac{\varepsilon_{1}}{\omega_{T O^{-}}^{2} \omega^{2}+i \Gamma \omega},
$$

where $\omega_{\mathrm{TO}}$ and $\Gamma$ are the TO mode frequency and the damping factor, respectively. The results of low and high damping factors are shown in Figs. 1(a) and (b), respectively.

(a)

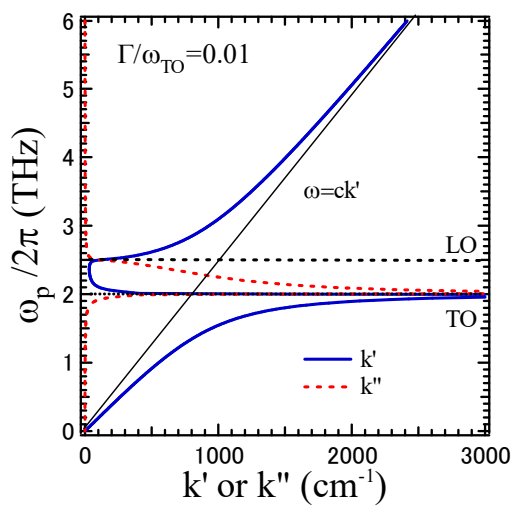

(b)

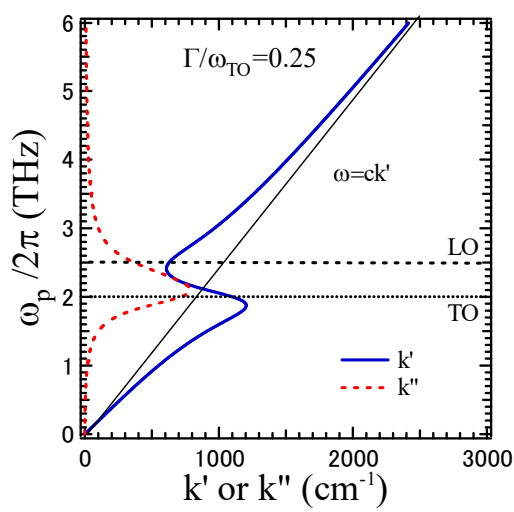

FIGURE 1. The complex dispersion relation of phonon polariton with a TO mode for (a) $\Gamma / \omega_{\mathrm{TO}}=0.01$, and (b) $\Gamma / \omega_{\mathrm{TO}}=0.25$, where $\Gamma$ and $\omega_{\mathrm{TO}}$ are damping factor and mode frequency of a TO mode, respectively.

\section{REAL AND IMAGINARY PARTS OF REFRACTIVE INDEX OF STRONTIUM}

\section{TITANATE}

The strontium titanate is a quantum paraelectric and does not undergo a ferroelectric phase transition down to $0 \mathrm{~K}$. The point symmetry of the paraelectric phase at room temperature is $\mathrm{Pm} 3 \mathrm{~m}$. The optical phonon modes of the strontium titanate at the $\Gamma$ point of the reciprocal lattice space is given by $3 \mathrm{~T}_{1 \mathrm{u}}+\mathrm{T}_{2 \mathrm{u}}$, where $3 \mathrm{~T}_{1 \mathrm{u}}$ modes are IR active and Raman inactive modes, and $\mathrm{T}_{2 \mathrm{u}}$ modes are IR inactive and silent modes. The $\mathrm{T}_{2 \mathrm{u}}$ modes were also studied hyper-Raman scattering by $\mathrm{K}$. Inoue et al.[16]. The $\mathrm{T}_{2 \mathrm{u}}$ modes were also studied by the traditional far-IR spectroscopy[17,18], and recently by THz-TDS [19]. However, the complex polariton dispersion was not yet reported.

The strontium titanate crystal was measured by broadband THz-TDS and the complex dielectric constants were determined [11]. First, the complex refractive index was calculated as shown in Fig. 2, in which the values observed by the transmission and reflection measurements are shown with the curves fitted by the damped harmonic oscillator (DHO) model [11]. The real and imaginary parts of the refractive index 
clearly show the sharp resonance at the lowest TO mode frequency near $=2.7 \mathrm{THz}$. Such behavior is very similar to that of a lithium tantalite crystal [20].

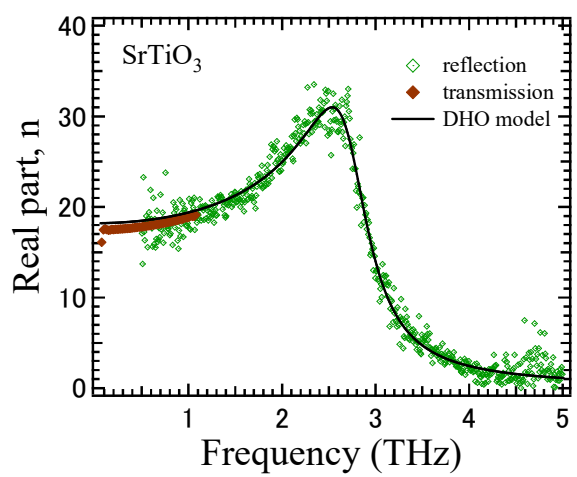

(a)

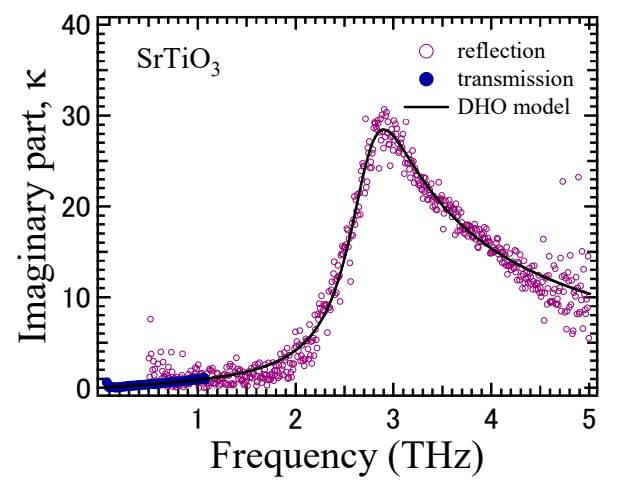

(b)

FIGURE 2. (a) Real and (b) imaginary parts of refractive index of a $\mathrm{SrTiO}_{3}$ crystal in the $\mathrm{THz}$ frequency range. Solid lines show the fitted curve of DHO model.

\section{COMPLEX POLARITON DISPERSION OF STRONTIUM TITANATE}

The complex polariton dispersion was calculated using the result of the complex dielectric constant. The dispersion relations for the real and imaginary parts of a wave vector of a strontium titanate crystal for $T_{1 u}$ mode calculated from observed values of complex refractive indices were shown in Figs. 3(a) and (b), respectively. The resonance was clearly observed in the vicinity of the lowest $\mathrm{TO}$ mode frequency $\omega_{\mathrm{TO}}=2.7 \mathrm{THz}$.

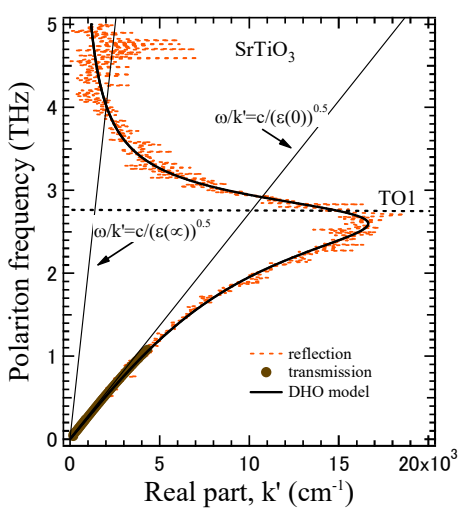

(a)

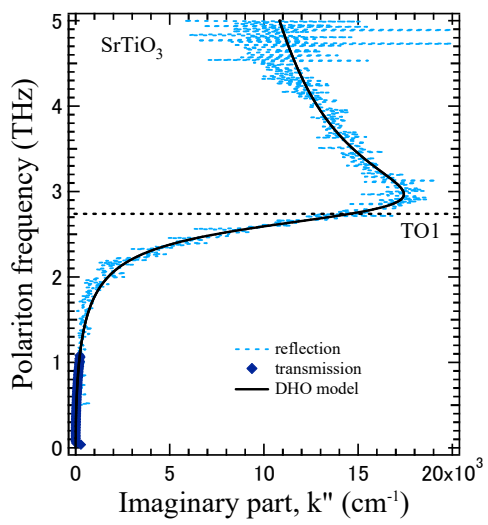

(b)

FIGURE 3. Polariton dispersion relations of the (a) real and (b) imaginary parts of the wavevector for the lowest-frequency $\mathrm{T}_{1 \mathrm{u}}$ symmetry polariton of a $\mathrm{SrTiO}_{3}$ crystal. 


\section{CONCLUSION}

The lowest frequency Raman inactive and IR active $\mathrm{T}_{1 \mathrm{u}}$ mode of a quantum paraelectric $\mathrm{SrTiO}_{3}$ crystal was studied by the broadband polarized Terahertz Time-Domain Spectroscopy using the coherent $\mathrm{THz}$ radiation from 0.2 to $5.0 \mathrm{THz}$. The dispersion relations of the real and imaginary parts of a polariton wavevector were determined from the observed real and imaginary parts of refractive indices for the $\mathrm{T}_{1 \mathrm{u}}$ symmetry phonon-polariton, respectively. The resonance in the vicinity of TO mode frequency was clearly observed. The observed complex dispersion relations of the $\mathrm{T}_{1 \mathrm{u}}$ symmetry are in agreement within the experimental uncertainty with the calculated dispersion curves by the damped harmonic oscillator model.

\section{ACKNOWLEDGEMENTS}

Authors are very thankful for the technical support on THz-TDS measurements to Sendai Office, Advantest Corporation. This work was partially supported by the Sasakawa Scientific Research Grant.

\section{REFERENCES}

1. Fano F: Atomic Theory of Electromagnetic Interactions in Dense Materials. Phys. Rev. 1956; 103: 1202-1218.

2. Born M and Huang K: Dynamical theory of crystal lattices. Oxford: Clarendon Press; 1956.

3. Henry CH and Hopfield JJ: Raman Scattering by Polaritons. Phys. Rev. Lett. 1965; 15: 964-966.

4. Barker, $\mathrm{J}_{\mathrm{R}}$. AS: Transverse and Longitudinal Optic Mode Study in $\mathrm{MgF}_{2}$ and $\mathrm{ZnF}_{2}$. Phys. Rev. 1964; 136: A1290-1295.

5. Claus R, Merten L, Brandmüller J: Light scattering by phonon-polaritons. Berlin Heidelberg: Springer-Verlag; 1975.

6. Müller KA and Burkard $\mathrm{H}: \mathrm{SrTiO}_{3}$ : An intrinsic quantum paraelectric below 4 K. Phys. Rev. B 1979; 19, 3593-3602.

7. Inoue K, Asai N, and Samejima T: Observation of the phonon polariton in the centrosymmetric crystal of $\mathrm{SrTiO}_{3}$ by Hyper Raman scattering. J. Phys. Soc. Jpn. 1980; 48: 1787-1788.

8. Shibuya T, Suizu K, and Kawase K: Widely Tunable Monochromatic Cherenkov Phase-Matched Terahertz Wave Generation from Bulk Lithium Niobate. Appl. Phys. Express.; 2010; 3: 082201-1-3.

9. Kojima S, Shibata T, Igawa H, Takayama H, Kim TH, and Mori T: Broadband terahertz time-domain spectroscopy: crystalline and glassy drug materials. IOP Conf. Series, Materials Science and Engineering (MSE). 2014; 54: 012001-1-6.

10. Kojima S, Tsumura N, Takeda MW, and Nishizawa S: Far-infrared phonon-polariton dispersion 
probed by terahertz time-domain spectroscopy. Phys. Rev. B. 2003; 67: 035102-1-5.

11. Mori T, Igawa H, and Kojima S: Progress of ultrafast terahertz time-domain spectroscopy: Raman inactive soft mode in quantum paraelectric $\mathrm{SrTiO}_{3}$, IOP Conf. Series, Materials Science and Engineering, 2014; 54, 012006-1-5.

12. Igawa $\mathrm{H}$, Mori $\mathrm{T}$, and Kojima $\mathrm{S}$ : Terahertz time-domain spectroscopy of congruent $\mathrm{LiNbO}_{3}$ and $\mathrm{LiTaO}_{3}$ crystals, Jpn. J. Appl. Phys., 2014; 53, 05FE01-1-4.

13. Kobayashi Y, Shibata T, Mori T, and Kojima S: Terahertz Time-Domain Spectroscopy and Low-Frequency Raman Scattering of Crystalline and Glassy Pharmaceutical Indapamide, Int. Lett. Chem. Phys. Astron., 2015, 46, 16-22.

14. Laughman L, Davis LW, and Nakamura T: Raman-Scattering Line Shape of the Soft E Polariton Mode in $\mathrm{BaTiO}_{3}$. Phys. Rev. B. 1972; 6: 3322-3326.

15. Tominaga $\mathrm{Y}$ and Nakamura T: Forward and backward Raman scattering from $\mathrm{KH}_{2} \mathrm{PO}_{4}$. Ferroelectrics. 1978; 21: 317-318.

16. Inoue K, Asai N, and Samejima T: Experimental Study of the Hyper-Raman Scattering Due to Raman Inactive Lattice Vibration in $\mathrm{SrTiO}_{3}$. J. Phys. Soc. Jpn. 1981; 50, 1291-1301.

17. Servoin JL, Luspin Y, and Gervais F: Phys. Rev. B 22, 5501 (1980).

18. Kanahira K, Hoshina $\mathrm{T}$, Takeda $\mathrm{H}$, and Tsurumi $\mathrm{T}$ : Measurement of ionic polarization of $\mathrm{SrTiO}_{3}$ single crystal by far-infrared spectroscopic ellipsometry. Appl. Phys. Lett. 2014; 105, 042901-1-3.

19. Matsumoto N, Fujii T, Kageyama K, Takagi H, Nagashima T, and Hangyo M: Measurement of the Soft-Mode Dispersion in $\mathrm{SrTiO}_{3}$ by Terahertz Time-Domain Spectroscopic Ellipsometry. Jpn. J. Appl. Phys. 2002; 48, 19KC11-1-4.

20. Kojima $\mathrm{S}$ and Mori $\mathrm{T}$ : Broadband terahertz time-domain spectroscopy of ferroelectric $\mathrm{LiTaO}_{3}$ : Phonon polariton dispersion. AIP Proceedings. 2014; 1627, 52-1-6. 